\title{
Ezetimibe in Combination With a Statin Does Not Reduce All-Cause Mortality
}

\author{
Akshar Y. Patel ${ }^{\mathrm{a}}$, Jayasree Pillarisetti ${ }^{\mathrm{b}}$, Joshua Marr ${ }^{\mathrm{b}}$, James L. Vacek ${ }^{\mathrm{b}, \mathrm{c}}$
}

\begin{abstract}
Background: Although the ezetimibe-statin combination has been shown to reduce LDL cholesterol by $12 \%$ compared to a statin alone, its effect on hard clinical endpoints such as mortality is less certain. Prior trials evaluated this combination in highly select population groups, but impact on all- cause mortality in the general population has not been reported.
\end{abstract}

Methods: A total of 3,827 subjects who were prescribed either a statin (group 1) or the combination of statin with ezetimibe (group 2) between January 1st, 2005 and January 1st, 2008 were studied. Socio-demographic and clinical variables and mortality records were analyzed. Univariate and stepwise multivariate logistic regression analysis was performed to identify the impact of ezetimibe on all-cause mortality, controlling for patient characteristics, selected cardiovascular diseases and risk factors, and medications.

Results: Group $1(\mathrm{n}=2,909)$, and group $2(\mathrm{n}=918)$ were similar in regards to most demographic variables, 152 patients died from any cause during the study period. There was no difference in all cause mortality between the groups. Hypertension, higher HDL-C and omega-3 fatty acid use were associated with ezetimibe use in this cohort of patients and were considered as covariates in the analysis. Patients on the drug combination did not experience lower mortality after controlling for covariates and other significant risk factors.

Conclusions: No significant mortality benefit was found with the use of ezetimibe in combination with a statin over use of a statin alone. Omega-3 fatty acid use and higher HDL-C demonstrated a substantial survival benefit.

\footnotetext{
Manuscript accepted for publication April 8, 2013

${ }^{a}$ Department of Medicine, Washington University School of Medicine, St. Louis, MO, USA

${ }^{\mathrm{b}}$ Department of Internal Medicine, University of Kansas School of Medicine and Medical Center, Kansas City, KS, USA

${ }^{\mathrm{c} C}$ Corresponding author: James L. Vacek, Mid America Cardiology at The University of Kansas Hospital, 3901 Rainbow Blvd, Kansas City, KS 66160, USA. Email: jlvacek@kumc.edu
}

doi: http://dx.doi.org/10.4021/jocmr1371w
Keywords: Ezetimibe; LDL; HDL; Mortality

\section{Introduction}

Ezetimibe is a cholesterol lowering medication that works by blocking enteric and biliary absorption of cholesterol, although its exact mechanism of action and affect on various other body systems related to atherosclerosis are under debate [1]. Approved in 2002, Ezetimibe in combination with a statin has been shown to reduce LDL cholesterol ((LDL-C)) $15 \%$ more compared to a statin taken alone [2]. Ezetimibe as a monotherapy has been shown to reduce LDL-C by 18 $\%$ when compared to a placebo $[2,3]$. Because the association between LDL-cholesterol reduction and decreased cardiovascular risk has been clearly established, ezetimibe was assumed to possess clinical efficacy and became routinely prescribed [4].

Although the effectiveness of ezetimibe in lowering cholesterol has been demonstrated in several studies, its clinical efficacy has been called into question following the ENHANCE trial (Ezetimibe and Simvastatin in Hypercholesterolemia Enhances Atherosclerosis Regression) which showed that there was no difference in the carotid intimamedia thickness in patients taking simvastatin and ezetimibe compared to those on simvastatin alone, despite having reduced overall total cholesterol, LDL-C, triglycerides , and C-reactive protein [5].

Other studies that followed yielded conflicting results with SANDS (Stop Atherosclerosis in Native Diabetics Study) and VYCTOR (Vytorin on Carotid Intima-Media Thickness and Overall Arterial Rigidity) demonstrating a reduction in carotid intima-media thickness. However the ARBITER 6 HALTS trial (Arterial Biology for the Investigation of the Treatment Effects of Reducing Cholesterol 6-HDL and LDL Treatment Strategies in Atherosclerosis) showed no improvement in intima-media thickness with the addition of ezetimibe. Further, the impact of ezetimibe on hard clinical endpoints such as mortality and cardiovascular events has not been proven till date [6-8]. Two studies 


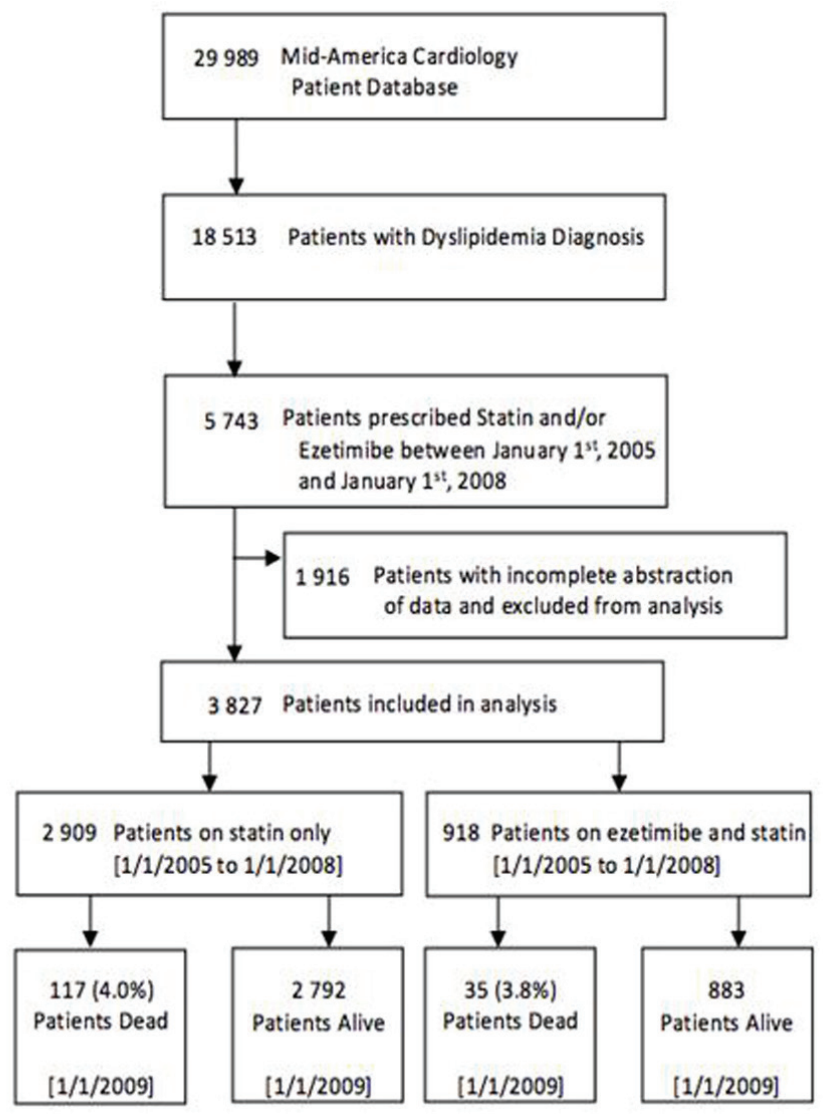

Figure I. Flowchart of cohort assembly.

(SEAS: Simvastatin and Ezetimibe in Aortic Stenosis, and SHARP: Study of Heart and Renal Protection) that demonstrated reduction in major cardiovascular events involved comparison of patients taking a combination of a statin plus ezetimibe versus those taking a placebo alone [9, 10]. The true clinical effect of ezetimibe can only be inferred by using a comparison group that takes a statin alone.

We therefore conducted a retrospective cohort study to compare differences in all-cause mortality between patients taking ezetimibe and a statin, and those on monotherapy with a statin alone.

\section{Methods}

A flowchart of cohort assembly can be found in Figure 1. The University of Kansas Hospital Mid-America Cardiology database of electronic patient medical records was used for this study. The study was carried out after obtaining approval from the human subjects committee and waiver of consent for data retrieval.

All social, demographic and clinical variables were obtained from the electronic database. Baseline lipid values of all patients on a statin prior to initiation of ezetimibe were also retrieved.

Study population; Patients who were not diagnosed with dyslipidemia were excluded, and only patients who were prescribed a statin were included. Patients taking combination ezetimibe and a statin (group 1) were compared to those prescribed only a statin (group 2). In order to account for differences in prescribing patterns due to the novelty of ezetimibe, patients who were prescribed either medication before January 1st 2005 were excluded. There was a dramatic fall in ezetimibe prescriptions after articles with negative and inconclusive findings were published, therefore new prescriptions after January 1st, 2008 were also excluded [5].

Follow-up: All patients were followed until January 2009. Patients who were on either medication for at least 1 year and died during this time period were noted.

\section{Endpoint}

The study's primary endpoint was all cause mortality.

\section{Statistical analysis}

Continuous variables between the groups were compared using unpaired t tests or Wilcoxon two-sample tests depending 
Table 1. Characteristics of Ezetimibe Plus Statin Versus Statin Only Patients

\begin{tabular}{|c|c|c|c|}
\hline Characteristic & Statin and Ezetimibe $(n=918)$ & Statin only $(n=2,909)$ & P-value \\
\hline Gender (Male) & $517(56 \%)$ & $1,596(55 \%)$ & 0.447 \\
\hline Age & $62.1 \pm 11.5$ & $63.6 \pm 12.3$ & 0.001 \\
\hline BMI & $31.5 \pm 6.9$ & $31.2 \pm 7.6$ & 0.413 \\
\hline Weight & $203.7 \pm 50.8$ & $200.7 \pm 51.3$ & 0.119 \\
\hline History of Tobacco use & $517(56 \%)$ & $1,636(56 \%)$ & 0.970 \\
\hline Cholesterol & $170.1 \pm 62.3$ & $169.6 \pm 46.3$ & 0.785 \\
\hline LDL-C & $96.2 \pm 42.1$ & $96.6 \pm 40.3$ & 0.116 \\
\hline HDL-C & $47.1 \pm 13.3$ & $48.2 \pm 14.4$ & 0.050 \\
\hline Hypertension & $600(65 \%)$ & $2,051(71 \%)$ & 0.004 \\
\hline Coronary Artery Disease & $405(44 \%)$ & $1,027(35 \%)$ & $<0.001$ \\
\hline Diabetes & $250(27 \%)$ & $838(29 \%)$ & 0.378 \\
\hline Myocardial Infarction & $47(5 \%)$ & $160(6 \%)$ & 0.738 \\
\hline $\mathrm{CABG}$ & $60(7 \%)$ & $131(5 \%)$ & 0.019 \\
\hline PCI & $91(10 \%)$ & $245(8 \%)$ & 0.181 \\
\hline Cerebrovascular Accident & $42(5 \%)$ & $161(6 \%)$ & 0.273 \\
\hline Transient Ischemia Attack & $34(4 \%)$ & $120(4 \%)$ & 0.630 \\
\hline Death & $35(4 \%)$ & $117(4 \%)$ & 0.652 \\
\hline Beta-blocker & $605(66 \%)$ & $1,838(63 \%)$ & 0.145 \\
\hline ACE-I & $473(52 \%)$ & $1,584(54 \%)$ & 0.129 \\
\hline $\mathrm{ARB}$ & $185(20 \%)$ & $547(19 \%)$ & 0.361 \\
\hline Omega-3 & $378(41 \%)$ & $935(32 \%)$ & $<0.001$ \\
\hline Aspirin & $722(79 \%)$ & $2,165(74 \%)$ & 0.009 \\
\hline Diuretic & $411(45 \%)$ & $1,319(45 \%)$ & 0.790 \\
\hline
\end{tabular}

ACE-I: angiotensin converting enzyme inhibitor; ARB: angiotensin II receptor blocker; BMI: body mass index; CABG: coronary artery bypass graft; HDL-C: high density lipoprotein cholesterol; LDL-C: low density lipoprotein cholesterol; $\mathrm{PCl}$ : percutaneous coronary intervention.

on whether the variables were normally distributed, and categorical variables were compared using two-sided chi square tests. Characteristics between patients prescribed or not prescribed ezetimibe are compared using the same methods. Odds ratios were calculated using logistic regression and 95\% confidence intervals. Logistic regression modeling was done to determine whether measured variables predicted if a patient had greater odds of being prescribed ezetimibe, and stepwise logistic regression was used to determine the opti- mal model. Odds of mortality were then calculated for each variable using logistic regression. All variables were put into a full model to control for confounding, and stepwise regression was used to determine the optimal model Statistical analysis of the data was performed using SAS version 9.1.3 (SAS Inc, North Carolina, USA). Statistical significance was considered present when $\mathrm{P}<0.05$.

The authors of this manuscript have certified that they comply with the Principles of Ethical Publishing in the Inter- 
Table 2. Odds of Being Prescribed Ezetimibe

\begin{tabular}{lll}
\hline Characteristic & Unadjusted Odds Ratio & Stepwise Model \\
\hline HDL-C & $0.994(0.989-0.999)$ & $0.992(0.985-1.000)$ \\
Hypertension & $0.805(0.705-0.919)$ & $0.758(0.609-0.942)$ \\
Omega-3 fatty acids & $1.689(1.483-1.923)$ & $1.457(1.178-1.802)$ \\
\hline
\end{tabular}

HDL-C: high density lipoprotein cholesterol.

national Journal of Cardiology [11].

\section{Results}

There were some significant differences between the groups (Table 1). The ezetimibe plus statin group was younger $(\mathrm{P}<$ $0.001)$ and had lower HDL-C levels $(P=0.050)$ with higher incidence of coronary artery disease $(\mathrm{P}<0.001)$ and $\mathrm{CABG}$ procedures $(\mathrm{P}=0.019)$ in comparison to the statin only group. Hypertension was more prevalent in the statin-only group $(\mathrm{P}=0.004)$. There was no difference between the total cholesterol $(\mathrm{P}=0.785)$ or LDL $(\mathrm{P}=0.116)$ between the two groups at baseline.

Logistic regression was used to determine whether certain characteristics increased the odds of a patient being prescribed ezetimibe (Table 2). Using stepwise regression, three variables significantly changed the odds. Patients with higher HDL levels had lower odds of being on ezetimibe in addition to a statin. Patients diagnosed with hypertension were $19.5 \%$ less likely to be on both medications, and those taking omega- 3 were $69 \%$ more likely to be taking ezetimibe.

The odds of mortality based on the measured demographic variables, cholesterol levels, disease diagnoses, cardiovascular procedures, and cardiovascular medications were each calculated independently using logistic regression (Table 3). No survival advantage was seen with ezetimibe added to a statin (4\% in both groups). When controlling for all measured factors, advancing age, a history of smoking, hypertension, a lower HDL level, having diabetes, taking beta-blockers, taking diuretics, and not taking omega- 3 fatty acids were all significantly associated with mortality. Ezetimibe dropped out of the final model and was then added back to find a final odds ratio. As could be predicted from looking at Table 1, a patient who takes both ezetimibe and statins was not found to benefit with regard to all cause mortality from a patient taking only statins (OR 1.067, 95\% CI: $0.713-1.598)$. Omega-3 fatty acid use (OR $0.554,95 \% \mathrm{CI}$ : $0.373-0.823$ ) or a higher HDL-C (OR $0.974,95 \%$ CI: 0.959 - 0.989) emerged as significantly protective of all cause mortality.

\section{Discussion}

This study adds to the growing body of evidence that ezetimibe may not provide substantial outcomes benefit in the treatment of hyperlipidemia. Our data indicate that there is no apparent mortality benefit for ezetimibe over mid-term follow up after one to three years of use.

Two trials where ezetimibe has been shown to have clinical efficacy are the SEARS and the SHARP trial. In the SEAS trial (Simvastatin and Ezetimibe in Aortic Stenosis) which compared patients on simvastatin $40 \mathrm{mg}$ and ezetimibe $10 \mathrm{mg}$ to those on placebo, fatal and nonfatal myocardial infarctions were significantly reduced with associated reductions in LDL-C. The SHARP trial demonstrated a $17 \%$ reduction in major atherosclerotic events. However, both these trials involve use of a statin and ezetimibe in comparison with a placebo and the observed clinical benefits could be due to the statin alone $[9,10]$. In contrast our study compares the mortality benefit in patients taking statin and ezetimibe against those taking statin alone. To our knowledge ours is the first study that attempts to look at the clinical efficacy of ezetimibe in comparison to a statin alone and demonstrated no additive benefit for using ezetimibe.

Ezetimibe works by inhibiting the cholesterol absorption in the jejunum while statins work by inhibiting cholesterol synthesis by inhibiting the enzyme HMG CoA reductase [12]. Reduced hepatic synthesis of LDL-C by the statins results in up regulation of hepatic LDL receptors and consequent increase in uptake of LDL-C from the blood resulting in reduced serum LDL levels [13]. However, these effects also result in enhanced cholesterol absorption. Thus ezetimibe which acts by inhibiting cholesterol absorption in the jejunum is supposed to have a complementary mechanism of action to that of the statins and the combination has been shown to achieve LDL-C reductions that have not been possible with statin monotherapy alone. However, these reductions in LDL-C have failed to demonstrate clinical benefits as seen in the ENHANCE and the ARBITER 6 HALTS trials $[5,8]$. Thus the randomized control trial (IMPROVE IT) which is testing clinical events in acute coronary syndrome patients on ezetimibe in addition to intensive statin treatment 
Table 3. Odds of Mortality Based on Cardiovascular Risk Factors

\begin{tabular}{|c|c|c|c|}
\hline Characteristic & Unadjusted Odds Ratio & Adjusted Odds Ratio & Stepwise Model \\
\hline Gender (Male) & $1.329(0.952-1.856)$ & $1.041(0.672-1.613)$ & - \\
\hline Age & $1.063(1.046-1.080)$ & $1.066(1.050-1.083)$ & $1.059(1.042-1.077)$ \\
\hline BMI & $0.993(0.953-1.034)$ & $0.979(0.933-1.026)$ & - \\
\hline Weight & $0.995(0.989-1.001)$ & $0.999(0.992-1.007)$ & - \\
\hline History of Smoking & $1.956(1.370-2.794)$ & $1.929(1.337-2.784)$ & $1.884(1.303-2.725)$ \\
\hline Cholesterol & $0.992(0.986-0.997)$ & $0.995(0.989-1.001)$ & - \\
\hline LDL-C & $1.004(0.999-1.009)$ & $1.006(1.001-1.010)$ & $1.005(1.001-1.010)$ \\
\hline HDL-C & $0.969(0.955-0.984)$ & $0.976(0.960-0.992)$ & $0.974(0.959-0.989)$ \\
\hline Hypertension & $1.057(0.741-1.508)$ & $0.643(0.435-0.951)$ & $0.840(0.583-1.211)^{*}$ \\
\hline Coronary Artery Disease & $2.074(1.497-2.874)$ & $1.308(0.878-1.948)$ & - \\
\hline Diabetes & $2.490(1.797-3.450)$ & $2.319(1.653-3.252)$ & $2.535(1.773-3.624)$ \\
\hline Myocardial Infarction & $1.105(0.555-2.200)$ & $0.858(0.412-1.788)$ & - \\
\hline CABG & $3.719(1.624-4.552)$ & $1.501(0.847-2.659)$ & - \\
\hline PCI & $0.971(0.544-1.733)$ & $0.844(0.448-1.591)$ & - \\
\hline CVA & $3.194(1.281-3.760)$ & $1.197(0.673-2.126)$ & - \\
\hline TIA & $1.737(0.890-3.350)$ & $1.536(0.767-3.077)$ & - \\
\hline Beta-blocker & $2.274(1.524-3.394)$ & $1.676(1.109-2.535)$ & $1.625(1.064-2.482)$ \\
\hline ACE-I & $1.543(1.102-2.162)$ & $1.040(0.712-1.519)$ & - \\
\hline $\mathrm{ARB}$ & $1.328(0.906-1.948)$ & $1.149(0.760-1.737)$ & - \\
\hline Omega-3 & $0.561(0.382-0.823)$ & $0.547(0.369-0.811)$ & $0.554(0.373-0.823)$ \\
\hline Aspirin & $1.231(0.827-1.830)$ & $0.841(0.546-1.295)$ & - \\
\hline Diuretic & $2.020(1.447-2.820)$ & $1.407(0.961-2.059)$ & $1.461(1.011-2.112)$ \\
\hline Ezetimibe & $0.946(0.644-1.390)$ & $1.093(0.733-1.628)$ & $\begin{array}{l}1.067(0.713- \\
1.598)^{* *}\end{array}$ \\
\hline
\end{tabular}

ACE-I: angiotensin converting enzyme inhibitor; ARB: angiotensin II receptor blocker; BMI: body mass index; CABG: coronary artery bypass graft; CVA: cerebrovascular accident; HDL-C: high density lipoprotein cholesterol; LDL-C: low density lipoprotein cholesterol; PCI: percutaneous coronary intervention; TIA: transient ischemia attack; *Hypertension was added back into the model to control confounding; **Ezetimibe was added back into the stepwise model because it drops out. $P$ values $<0.05$ for all odds ratios by stepwise model except * and **.

is highly anticipated.

Limitations: Though this study indicates a lack of clinical efficacy for ezetimibe, it does face several limitations. Despite the large sample size, the data only comes from one group of cardiologists at one medical center and is retro- spective. Our study did not include lipid values following administration of ezetimibe. Thus the benefits of LDL reduction could not be demonstrated in our study. However the primary aim was to look at the clinical endpoint of mortality as LDL reduction by ezetimibe has already been well estab- 
lished.

\section{Conclusion}

No significant mortality benefit was found with the use of ezetimibe in combination with a statin. Further randomized trials investigating the clinical efficacy of ezetimibe in the general population are needed.

\section{Declaration}

All authors take responsibility for all aspects of the reliability and freedom from bias of the data and their discussed interpretation.

\section{Conflicts of interest}

The authors report no conflicts of interest.

\section{References}

1. Taylor AJ. Given The ENHANCE trial results, ezetimibe is still unproven. Cleveland Clinic J Med 2008; 75:497-506.

2. Morrone D, Weintraub WS, Toth PP, Hanson ME, Lowe RS, Lin J, Shah AK, et al. Lipid-altering efficacy of ezetimibe plus statin and statin monotherapy and identification of factors associated with treatment response: a pooled analysis of over 21,000 subjects from 27 clinical trials. Atherosclerosis. 2012;223(2):251-261.

3. Pandor A, Ara RM, Tumur I, Wilkinson AJ, Paisley S, Duenas A, Durrington PN, et al. Ezetimibe monotherapy for cholesterol lowering in 2,722 people: systematic review and meta-analysis of randomized controlled trials. J Intern Med. 2009;265(5):568-580.

4. Ballantyne CM, Houri J, Notarbartolo A, Melani L, Lipka LJ, Suresh R, Sun S, et al. Effect of ezetimibe coadministered with atorvastatin in 628 patients with primary hypercholesterolemia: a prospective, randomized, double-blind trial. Circulation. 2003;107(19):24092415.

5. Kastelein JJ, Akdim F, Stroes ES, Zwinderman AH, Bots ML, Stalenhoef AF, Visseren FL, et al. Simvastatin with or without ezetimibe in familial hypercholesterolemia. N Engl J Med. 2008;358(14):1431-1443.

6. Fleg JL, Mete M, Howard BV, Umans JG, Roman MJ, Ratner RE, Silverman A, et al. Effect of statins alone versus statins plus ezetimibe on carotid atherosclerosis in type 2 diabetes: the SANDS (Stop Atherosclerosis in Native Diabetics Study) trial. J Am Coll Cardiol. 2008;52(25):2198-2205.

7. Meaney A, Ceballos G, Asbun J, Solache G, Mendoza E, Vela A, Meaney E. The VYtorin on Carotid intimamedia thickness and overall arterial rigidity (VYCTOR) study. J Clin Pharmacol. 2009;49(7):838-847.

8. Villines TC, Stanek EJ, Devine PJ, Turco M, Miller M, Weissman NJ, Griffen L, et al. The ARBITER 6-HALTS Trial (Arterial Biology for the Investigation of the Treatment Effects of Reducing Cholesterol 6-HDL and LDL Treatment Strategies in Atherosclerosis): final results and the impact of medication adherence, dose, and treatment duration. J Am Coll Cardiol. 2010;55(24):2721-2726.

9. Rossebo AB, Pedersen TR, Boman K, Brudi P, Chambers JB, Egstrup K, Gerdts E, et al. Intensive lipid lowering with simvastatin and ezetimibe in aortic stenosis. $\mathrm{N}$ Engl J Med. 2008;359(13):1343-1356.

10. Baigent C, Landray MJ, Reith C, Emberson J, Wheeler DC, Tomson C, Wanner C, et al. The effects of lowering LDL cholesterol with simvastatin plus ezetimibe in patients with chronic kidney disease (Study of Heart and Renal Protection): a randomised placebo-controlled trial. Lancet. 2011;377(9784):2181-2192.

11. Coats AJ, Shewan LG. Statement on authorship and publishing ethics in the International Journal of Cardiology. Int J Cardiol. 2011;153(3):239-240.

12. Toth PP, Davidson MH. Cholesterol absorption blockade with ezetimibe. Curr Drug Targets Cardiovasc Haematol Disord. 2005;5(6):455-462.

13. Huff MW, Burnett JR. 3-Hydroxy-3-methylglutaryl coenzyme A reductase inhibitors and hepatic apolipoprotein B secretion. Curr Opin Lipidol. 1997;8(3):138-145. 